\title{
Diagnostik und Therapie in der Proktologie
}

Fried, M

DOI: https://doi.org/10.1007/s11377-010-0453-5

Other titles: Diagnostics and therapy in proctology

Posted at the Zurich Open Repository and Archive, University of Zurich ZORA URL: https://doi.org/10.5167/uzh-156452

Journal Article

Published Version

Originally published at:

Fried, M (2010). Diagnostik und Therapie in der Proktologie. Der Gastroenterologe, 5(4):307.

DOI: https://doi.org/10.1007/s11377-010-0453-5 
Gastroenterologe 2010 · 5:307

DOI 10.1007/s11377-010-0453-5

(c) Springer-Verlag 2010

\author{
M. Fried \\ Klinik für Gastroenterologie und Hepatologie, UniversitätsSpital Zürich
}

\section{Diagnostik und Therapie in der Proktologie}

\section{Interdisziplinäre Zusammenarbeit für eine optimale Behandlung der Patienten}

Proktologische Probleme gehören zum Alltag der ärztlichen Praxis und beschäftigen gleichermaßen den Internisten, den Gastroenterologen, den Dermatologen und den Chirurgen. Die interdisziplinäre Zusammenarbeit zwischen diesen Disziplinen steht im Vordergrund und ist Voraussetzung für eine optimale Behandlung des Patienten mit proktologischen Problemen. In dieser Ausgabe von „Der Gastroenterologe" werden die wichtigsten Themen der Proktologie von ausgewiesenen Experten abgehandelt und vor allem die Fortschritte auf diesem wichtigen klinischen Gebiet dargestellt.

Die Fistelchirurgie wurde in den letzten Jahren durch neue minimalinvasive Methoden wie den „Anal Fistula Plug“ bereichert, die eine Behandlung ohne ein Inkontinenzrisiko ermöglichen. Allerdings kann mit diesen Methoden nur in etwa der Hälfte der Fälle ein Fistelverschluss erreicht werden.

Besondere Fortschritte wurden in den letzten Jahren in der Erforschung der Pathogenese des Analkarzinoms erzielt. Die Häufigkeit dieses Tumors hat in den letzten Jahrzehnten stark zugenommen, insbesondere bei HIV-infizierten und immunsupprimierten Patienten. Die anale Infektion mit dem humanen Papillomavirus HPV wurde als hauptsächlicher Risikofaktor identifiziert und erklärt die Häufung dieses Tumors bei männlichen Homosexuellen. Das Analkarzinom entsteht aus einer analen, intraepithelialen Neoplasie, wobei die optimale Screeningmethode umstritten bleibt. Zur Verfügung stehen Verfahren wie eine anale Bürstenzytologie, eine High-Resolution-Anoskopie und eine HPV-Typisierung mittels Insitu-Hybridisierung. Der Stellenwert dieser Verfahren muss noch durch kontrollierte prospektive Studien evaluiert werden. Ebenfalls kontrovers ist die Therapie einer analen Dysplasie. So bleibt unklar, ob und in welchem Stadium der AIN eine Therapie, z. B. Imiquimod mit einer ablativen Therapie, sinnvoll ist. Andererseits herrscht weitgehender Konsens, dass ein Analkarzinom mit einer Radiochemotherapie behandelt werden sollte.

Hämorrhoidalleiden gehören $\mathrm{zu}$ den häufigsten Erkrankungen, entsprechend ist eine Vielzahl von Behandlungsmethoden entwickelt worden. Während nichtoperative Verfahren, wie u. a. die Sklerosierungstherapie eher in den Hintergrund getreten sind, hat sich die Staplerhämorrhoidopexie als Standardverfahren bei höhergradigen Hämorrhoiden etabliert. Die Erfahrungen mit neueren Verfahren wie z. B. der dopplergesteuerten Hämorrhoidenarterienligatur sind hingegen noch begrenzt.

\section{(2) Bei chronischen Analbeschwerden sollte immer an ein dermatologisches Grundleiden gedacht werden}

Besondere Probleme in der Praxis können ein Pruritus ani und ein Analschmerz ohne ein nachweisbares organisches Korrelat bereiten. Der psychogene Pruritus bzw. die Proctalgia fugax sind Ausschlussdiag- nosen, deren Behandlung sehr schwierig sein kann. Bei chronischen Analbeschwerden ist immer auch an ein dermatologisches Grundleiden zu denken: Viele dermatologische Erkrankungen können die Analregion befallen, was therapeutisch berücksichtigt werden sollte.

Die Beiträge vermitteln einen sehr guten Überblick über die aktuellen Entwicklungen und Fortschritte auf diesem wichtigen Gebiet. Besonderes Gewicht wird dabei auf die praktisch-klinische Seite der Proktologie gelegt; dem Kliniker werden damit wertvolle Hinweise für die Diagnostik und Therapie dieser Erkrankungen gegeben.

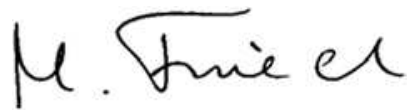

Michael Fried

\section{Korrespondenzadresse}

Prof. Dr. M. Fried

Klinik für Gastroenterologie und Hepatologie, UniversitätsSpital Zürich

Rämistr. 100, 8091 Zürich

Schweiz

michael.fried@usz.ch 